\title{
Application of virtual reality technology in practical teaching in Colleges
}

\author{
Cai Bao ${ }^{a}$, * \\ Engineering Training Center, Shanghai Polytechnic University, Shanghai, China \\ acaibao@sspu.edu.cn
}

Keywords: Practice Teaching; Virtual Reality Technology; Virtual simulation laboratory

Abstract: The content of practice teaching is generally characterized by danger and complexity, in particular, practical courses in mechanical and electronic circuits. Taking the practical course of machinery as an example, the feasibility of applying virtual reality technology in practical teaching is expounded, the advantages of various virtual reality devices in practical teaching are analyzed and compared, the main research results of school virtual simulation laboratory are briefly described, and the construction direction of virtual simulation for practical teaching in Colleges and universities is summarized.

\section{Introduction}

With the rapid development of computer technology, virtual reality (VR) has become a hot technology in recent years. It has four characteristics: immersion, interaction, imagination and mufti-perception. Virtual reality system is generally divided into desktop, immersive, distributed and enhanced according to different forms of user participation ${ }^{[1]}$.

Desktop virtual reality is simulated by personal computers and low-level workstations. Full interaction with virtual reality is achieved through external input devices, such as mouse, pen, etc. Desktop virtual reality is disturbed by the surrounding reality and reduces the participants' sense of reality.

Immersive virtual reality provides a completely immersive experience, giving the user a sense of being in the virtual world. It uses a helmet-mounted display to enclose participants 'vision, hearing and other sensations, and provides a new, virtual sensory space.

Distributed Virtual Reality (DVR) connects multiple users together through a computer network and participates in a virtual space at the same time, that is, multiple users can observe and operate the same virtual world through the network to achieve the purpose of collaborative work.

Augmented Reality Virtual Reality (AR-VR) can simulate the real world and simulate the real world by using virtual reality technology, and it can also enhance participants 'perception of the real environment.

\section{Application status of VR technology in education field}

Many scholars at home and abroad have studied the application of virtual reality in the field of education. Zhao et al. Used C\# programming language to develop a virtual reality system ${ }^{[2]}$. Kozik 
and others discussed the possibility of virtual simulation of MOOC course ${ }^{\text {[3-4]. DS Company }}$ applies DENEB software to optimize the virtual assembly production for general motors ${ }^{[5]}$. Patterson and others expounded the basic principles of 3D display ${ }^{[6]}$.

\section{Typical application results}

Due to the complexity and danger of operation in mechanical practice course, there are many simulation studies on practical teaching in engineering field in China. The following is a virtual reality practice course system developed by Shanghai Second Polytechnic University according to the traditional teaching contents of mechanical practice course.

1) ZSpace desktop virtual reality application: zSpace desktop virtual reality system is mainly used in the simulation study of single machine tool. The virtual simulation system mainly includes the study of machine tool structure, working principle and operation method. Fig. 1, the bottom-left corner of the virtual reality equipment in zSpace, to carry out the three-dimensional operation experience of machine tool, at the same time. In order to share the demonstration results, a nakedeye stereoscopic expansion system is developed, which can extend the operation screen of the teacher computer to the stereoscopic display and carry out on-site teaching. At present, this set of equipment has been used in daily teaching; students first carry out virtual simulation practice learning, and then enter the actual workshop for machine tool practice learning.

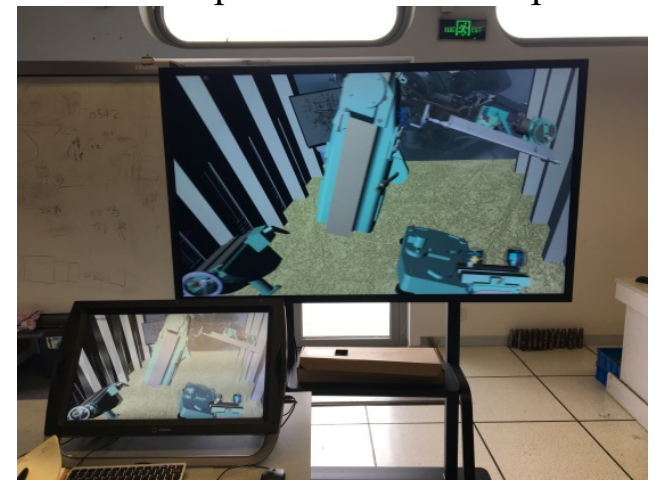

Fig. 1 desktop virtual reality system

2) HTC Helmet Immersion Virtual Reality Application: HTC Helmet is a mature product on the market; its biggest teaching advantage is the collaboration of many people. In the actual teaching, many students wear a helmet in the same virtual environment to coordinate the completion of the same learning task. As shown in Figure 2, three students completed the virtual assembly process learning in the virtual workshop.

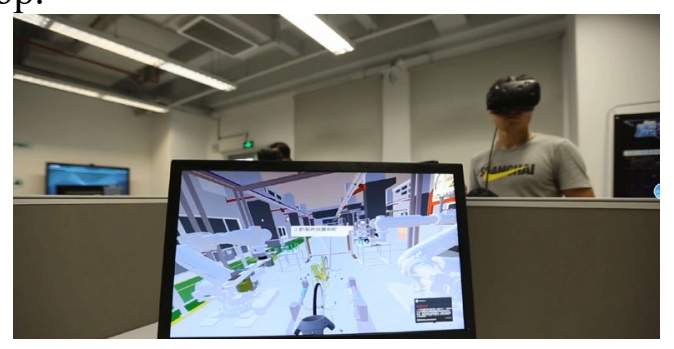

Fig. 2 immersive virtual reality system

3) Augmented Reality + Touch-Screen Virtual Reality Applications: The combination of mobile devices and large-screen touch-screen displays can achieve augmented reality sharing learning, increasing interaction and good display performance. As shown in Figure 3, the mobile end scan feature map for AR recognition, the large screen can display the identification content for classroom 
teaching, greatly enriching the practical teaching content.

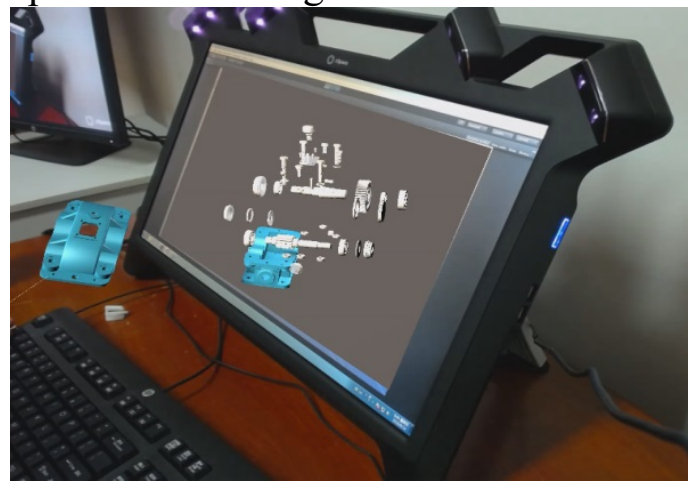

Fig. 3 augmented reality virtual reality system

4) Application of Holospace Distributed Virtual Reality System: Five-channel Distributed Virtual Reality System can be used for practical teaching of large-scale engineering production. Large-screen VR display system can be used to restore the production and manufacturing reality in $1: 1$. The system is composed of front screen, left screen and ground screen. The front screen and ground screen are controlled by two computers, and the left screen is composed of one. The computer is controlled separately, and the final stereoscopic picture is made up of five projectors.

As shown in Figure 4, Holospace Distributed Virtual Reality System can be used in the production time of intelligent manufacturing workshop. Because there is much production equipment in the workshop, it is not limited to the simulation of single equipment. The system simulation can be based on the workshop environment, which can make students experience the production practice more real and greatly increase the learning experience.

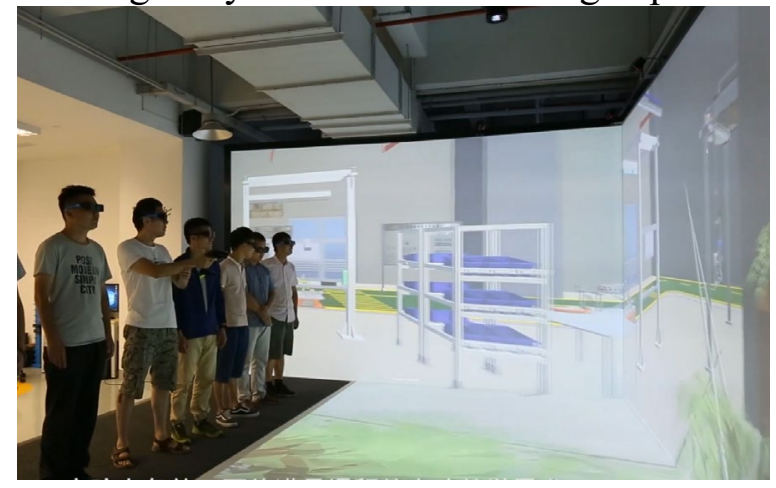

Fig. 4 distributed virtual reality system

In recent years, the development of virtual reality technology has shown a trend of blowout. A large number of virtual reality devices are used in all fields except games. At present, the development of virtual reality devices is mainly supported by Unity3D software [10]. The technical difficulty of development is greatly reduced. The content of development can be made according to the specific professional needs of the school.

\section{Analysis of the construction of Virtual Simulation Laboratory}

This paper analyzes the characteristics of laboratory construction, the advantages of virtual technology and the construction of economic cost.

(1) Super versatility, virtual reality technology as a key direction in the field of virtual simulation, technology itself has developed more mature, can be used in various industries, especially in the actual operation is difficult to achieve dangerous situation. 
(2) Good hierarchy of the system, the use of virtual reality technology in simulation teaching, production content can be divided into modules according to the professional teaching, difficult degree can be well distinguished, especially the professional basic courses and general basic courses teaching content can be hierarchical teaching.

(3) Good economic and constructive, at present the cost of mature virtual reality equipment varies greatly, HTC immersion virtual reality only a few thousand yuan, augmented reality + touch screen virtual reality construction costs tens of thousands of yuan, zSpace desktop virtual reality construction costs hundreds of thousands of yuan, and distributed virtual reality as many as millions of dollars In actual operation, it is necessary to decide according to school conditions and funds.

\section{Acknowledgements}

Foundation: Science and Technology Development Fund Project of Shanghai Polytechnic University "Engineering Training Course Development Based on Virtual Reality Technology" (Foundation No. EGD17XQD41).

\section{References}

[1] Okita A, Learning C\# programming with Unity 3D[J]. English Journal, 2015(1): 249-267.

[2] Zhao J, Gong Q M, Eisensten Z. Tunneling through a frequently changing and mixed ground: A case history in Singapore[J]. Tunneling and Underground Space Technology, 2007, 22 (4): $388-400$.

[3] Kozik T, Simon M. Preparing and managing the remote experiment in education [C].15th International Conference on Interactive Collaborative Learning. Villach: IEEE, 2012: 26-28.

[4] Joao B C. Virtual laboratories and M-learning: learning with mobile devices[C]. Proceedings of International Milti-Conference on Society, Cybernetics and Informatics, New York: IIIS, 2007: 275-278.

[5] Harel Beit-On. Delmia-Tecnomatix-The Duel for the Digital Factory [J]. PROMISING MARKET, $2002,5$.

[6] Patterson R. Human factors of stereo displays: An update. Journal of the Society for Information Display,2009,17(12):987-996. 